\title{
Prognostic role of neutrophil-to- lymphocyte ratio in breast cancer: a systematic review and meta-analysis
}

\author{
Josee-Lyne Ethier ${ }^{1,2}$, Danielle Desautels ${ }^{3}$, Arnoud Templeton ${ }^{4}$, Prakesh S. Shah ${ }^{5,6}$ and Eitan Amir ${ }^{1,2^{*}}$
}

\begin{abstract}
Background: The presence of a high neutrophil-to-lymphocyte ratio (NLR) has been associated with increased mortality in several malignancies. Here, we quantify the effect of NLR on survival in patients with breast cancer, and examine the effect of clinicopathologic factors on its prognostic value.

Methods: A systematic search of electronic databases was conducted to identify publications exploring the association of blood NLR (measured pre treatment) and overall survival (OS) and disease-free survival (DFS) among patients with breast cancer. Data from studies reporting a hazard ratio (HR) and 95\% confidence interval (Cl) or a $P$ value were pooled in a meta-analysis. Pooled HRs were computed and weighted using generic inverse variance. Meta-regression was performed to evaluate the influence of clinicopathologic factors such as age, disease stage, tumor grade, nodal involvement, receptor status, and NLR cutoff on the HR for OS and DFS. All statistical tests were two-sided.

Results: Fifteen studies comprising a total of 8563 patients were included. The studies used different cutoff values to classify high NLR (range 1.9-5.0). The median cutoff value for high NLR used in these studies was 3.0 amongst 13 studies reporting a HR for OS, and 2.5 in 10 studies reporting DFS outcomes. NLR greater than the cutoff value was associated with worse OS (HR 2.56, 95\% Cl=1.96-3.35; $P<0.001)$ and DFS ( $H R 1.74,95 \% \mathrm{Cl}=1.47-2.07 ; P<0.001)$. This association was similar in studies including only early-stage disease and those comprising patients with both early-stage and metastatic disease. Estrogen receptor (ER) and HER-2 appeared to modify the effect of NLR on DFS, because NLR had greater prognostic value for DFS in ER-negative and HER2-negative breast cancer. No subgroup showed an influence on the association between NLR and OS.

Conclusions: High NLR is associated with an adverse OS and DFS in patients with breast cancer with a greater effect on disease-specific outcome in ER and HER2-negative disease. NLR is an easily accessible prognostic marker, and its addition to established risk prediction models warrants further investigation.
\end{abstract}

Keywords: Breast cancer, Neutrophil-to-lymphocyte ratio, Prognosis, Disease-free survival, Overall survival, Meta-analysis, Systematic review

\section{Background}

The short-term and long-term prognosis of breast cancer depends on patient and tumor factors such as age, disease stage, and biological factors such as grade and receptor status. However, the behavior of breast cancer is unpredictable, with markedly different clinical outcomes seen

\footnotetext{
* Correspondence: eitan.amir@uhn.ca

${ }^{1}$ Division of Medical Oncology and Hematology, Princess Margaret Cancer Centre, 610 University Avenue 5-124, Toronto, ON M5G 2M9, Canada

${ }^{2}$ Department of Medicine, University of Toronto, Toronto, Canada

Full list of author information is available at the end of the article
}

even amongst patients with similar classical prognostic factors [1].

Inflammatory cells and mediators in the tumor microenvironment are thought to play an important role in cancer progression, and may account for some of this variability [2]. The presence of an elevated peripheral neutrophil-to-lymphocyte (NLR) ratio, an indicator of systemic inflammation, has been recognized as a poor prognostic factor in various cancers [3]. In a previous metaanalysis of 100 studies of patients with unselected solid tumors, increased NLR was associated with decreased 
overall survival (OS) (hazard ratio (HR) 1.81; 95\% confidence interval $(\mathrm{CI})=1.67-1.97 ; P<0.001)$ [4]. This effect was observed in all disease sites, subgroups, and stages. However, this study was not specific to breast cancer, and did not examine the impact of prognostic factors such as estrogen receptor (ER) or progesterone receptor (PR) status, HER2 status, disease stage, or menopausal status.

The aim of this study was to quantify the effect of peripheral blood NLR on OS and disease-free survival (DFS) in adult women with invasive breast cancer. We also examined the effect of clinicopathologic factors on the prognostic value of NLR.

\section{Methods}

\section{Data sources and searches}

This analysis was reported in accordance with the Preferred Reporting Items for Systematic Reviews and Meta-Analyses (PRISMA) guidelines [5]. The search strategy developed by Templeton et al. [4] was used with the addition of "breast neoplasms" and synonymous breast cancer-specific terms. An electronic search of the following databases was performed: Medline (host: OVID), Medline in Process, Medline Epub Ahead of Print (host: OVID), EMBASE (host: OVID), and Cochrane Database of Systematic Reviews. All databases were searched from January 2013 to April 2016, supplementing the initial systematic review that searched databases until different time points in 2013. Citation lists of retrieved articles were screened manually to ensure sensitivity of the search strategy. The full search strategy is described in Table 3 in Appendix 1.

\section{Study selection}

In order to reduce clinical heterogeneity, the following eligibility criteria were utilized: studies of adult women with breast cancer reporting on the prognostic impact of the peripheral blood NLR, where NLR was treated as a categorical variable; NLR collected prior to all treatment (surgery and/or systemic therapy); reporting of a multivariable $H R$ for OS, and/or DFS or progression-free survival (PFS), and corresponding 95\% CI and/or $P$ value; available as a full-text publication; clinical trials, cohort studies, or case-control studies; and English-language publication. Case reports, conference proceedings, and letters to editors were excluded. Corresponding authors were contacted to clarify missing or ambiguous data. When multiple publications or data analyses were available from the same dataset and if clarification on potentially duplicate data could not be obtained, the study reporting the larger number of patients was retained and other studies were excluded. Studies only presenting data in graphic form without reporting a numerical value for HR were excluded. All titles identified by the search were evaluated, and all potentially relevant publications were retrieved in full. Two reviewers (JE and DD) independently reviewed full articles for eligibility based on inclusion criteria and data extraction, and disagreements were resolved by consensus. Three relevant articles identified in the previous systematic review were also included [4].

\section{Data extraction}

The following details were extracted from included studies using predesigned data abstraction forms: name of first author, year of publication, journal, number of patients included in analysis, median age, disease stage (nonmetastatic, metastatic, mixed (nonmetastatic and metastatic)), collection of data (prospective, retrospective), cutoff value used to define high NLR, number of patients with each breast cancer subtype, number of premenopausal and postmenopausal patients, and HRs and associated 95\% CIs for OS, PFS, or DFS. Where more than one multivariable model was reported, HRs were extracted from models including the most participants.

\section{Risk of bias assessment}

Validity of included studies was assessed by two independent reviewers (J-LE and DD) using the Quality in Prognostic Studies (QUIPS) tool as described previously [6]. The QUIPS tool comprises 30 questions categorized into six domains (study participation, study attrition, prognostic factor measurement, outcome measurement, study confounding, and statistical analysis and reporting). Studies were rated according to each domain as being at low, moderate, or high risk of bias, based on the likelihood that they might alter the relationship between the prognostic factor and outcome.

\section{Statistical analyses}

Extracted data were pooled using RevMan 5.3 analysis software (Cochrane Collaboration, Copenhagen, Denmark). A meta-analysis was conducted for all included studies for each of the endpoints of interest if appropriate when clinical heterogeneity was minimal. The primary outcome of interest was OS, and intermediate endpoints such as PFS and/or DFS were secondary outcomes. Estimates for HRs were pooled and weighted by generic inverse variance, and were computed by fixed-effects or random-effects modeling. Heterogeneity was assessed using Cochran $Q$ and $I^{2}$ statistics. If significant heterogeneity was present $\left(I^{2}>50 \%\right.$ or Cochran $Q<0.1$ ), a random-effects model was used. Predefined subgroup analyses were conducted for disease stage (early, metastatic, mixed) using methods described by Deeks et al. [7] Meta-regression was performed to evaluate the effects of NLR cutoff, proportion of ER-positive patients, proportion of HER2-positive patients, proportion of triple-negative patients, median age, proportion of premenopausal patients, and proportion of patients with metastatic 
disease on the HR for OS and DFS. Meta-regression comprised a univariable linear regression weighted by individual study inverse variance and was performed using SPSS version 24 (IBM Corp, Armonk, NY, USA). A post-hoc meta-regression analysis testing the association between median duration of follow-up and the prognostic value of NLR was also performed. Multivariable meta-regression was not performed due to the small number of eligible studies leading to an undesirable risk of over-fitting. Publication bias was assessed by inspecting funnel plots visually. All statistical tests were two-sided, and statistical significance was defined as $P<0.05$.

\section{Results}

Fifteen studies comprising a total of 8563 patients were included (Fig. 1). Characteristics of included studies are described in Table 1, and further details are included in Table 4 in Appendix 2. All studies collected data retrospectively, and all were published in 2012 or later. Ten studies included only patients with earlystage breast cancer, while five included both early and metastatic disease.

\section{Overall survival}

Thirteen studies comprising a total of 8015 patients reported adjusted HRs for OS. The median cutoff value for high NLR was 3.0 (range 2.0-5.0). Median follow-up was reported in 11 studies, and ranged from 1.8 to 7.2 years (mean 4.69 years) (Table 4 in Appendix 2). Overall, a NLR greater than the cutoff value was associated with worse OS (HR 2.56, 95\% CI $=1.96-3.35 ; P<$ 0.001; see Fig. 2). There was statistically significant heterogeneity (Cochran $Q=0.009, I^{2}=55 \%$ ). This seems to be largely influenced by one study which showed a large effect size [8]. However, the association between NLR and OS was maintained in a sensitivity analysis omitting

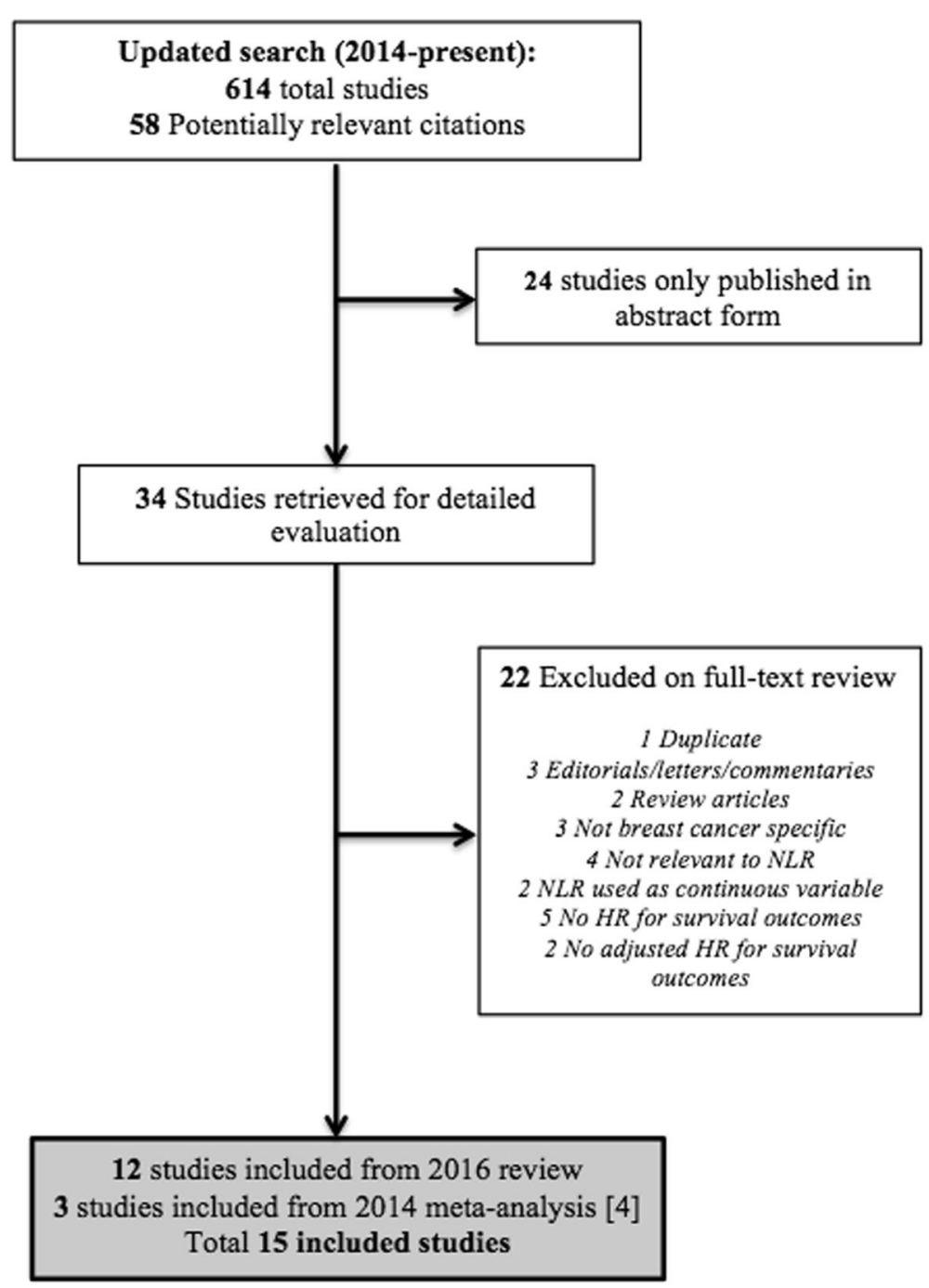

Fig. 1 Flow chart of study selection process. HR hazard ratio, NLR neutrophil-to-lymphocyte 
Table 1 Characteristics of included studies

\begin{tabular}{|c|c|c|c|c|}
\hline Study & Year & $\begin{array}{l}\text { Number of } \\
\text { patients }\end{array}$ & $\begin{array}{l}\text { Disease } \\
\text { stage }\end{array}$ & $\begin{array}{l}\text { NLR cutoff } \\
\text { value }\end{array}$ \\
\hline \multicolumn{5}{|l|}{ Overall survival } \\
\hline Azab et al. [23] ${ }^{a}$ & 2012 & 316 & Mixed & 3.3 \\
\hline Azab et al. [13] ${ }^{a}$ & 2013 & 437 & Mixed & 3.3 \\
\hline Bozkurt et al. [24] & 2015 & 85 & Early & 2.0 \\
\hline Dirican et al. [25] & 2015 & 1527 & Mixed & 4.0 \\
\hline Forget et al. [10] & 2014 & 720 & Early & 3.3 \\
\hline Jia et al. [14] & 2015 & 1570 & Early & 2.0 \\
\hline Koh et al. [8] & 2014 & 157 & Early & 2.3 \\
\hline Koh et al. [15] & 2015 & 1435 & Mixed & 5.0 \\
\hline Nakano et al. [9] & 2015 & 167 & Early & 2.5 \\
\hline Noh et al. [26] ${ }^{a}$ & 2013 & 442 & Early & 2.5 \\
\hline Pistelli et al. [27] & 2015 & 90 & Early & 3.0 \\
\hline Rimando et al. [28] & 2016 & 461 & Mixed & 3.8 \\
\hline Yao et al. [11] & 2014 & 608 & Early & 2.6 \\
\hline \multicolumn{5}{|l|}{ Disease-free survival } \\
\hline Asano et al. [12] & 2016 & 61 & Early & 3.0 \\
\hline Bozkurt et al. [24] & 2015 & 85 & Early & 2.0 \\
\hline Dirican et al. [25] & 2015 & 1527 & Mixed & 4.0 \\
\hline Forget et al. [10] & 2014 & 720 & Early & 3.3 \\
\hline Hong et al. [29] & 2015 & 487 & Early & 1.9 \\
\hline Jia et al. [14] & 2015 & 1570 & Early & 2.0 \\
\hline Koh et al. [8] & 2014 & 157 & Early & 2.3 \\
\hline Nakano et al. [9] & 2015 & 167 & Early & 2.5 \\
\hline Pistelli et al. [27] & 2015 & 90 & Early & 3.0 \\
\hline
\end{tabular}

NLR neutrophil-to-lymphocyte

ancluded in previous meta-analysis [4]

this study (HR 2.42, 95\% $\mathrm{CI}=1.89-3.09 ; \quad P<0.001$; Cochran $Q=0.03, I^{2}=48 \%$ ), although statistically significant heterogeneity remained.

Exploratory analysis identified breast cancer stage as an important source of heterogeneity. Subgroup analysis showed that the association between NLR and OS was maintained in studies including only early-stage disease, as well as those comprised of patients with both early and metastatic disease (HR 2.98 vs 2.30 respectively; $P$ for subgroup differences $=0.36$ ). There was no statistical heterogeneity when the study driving heterogeneity in the main analysis [8] was omitted from the early stage subgroup (Cochran $Q=0.28, I^{2}=20 \%$ ). Additionally, the effect of NLR on OS was retained (HR 2.56, 95\% CI = $1.82-3.60 ; P<0.001)$. Statistical heterogeneity remained among studies with mixed early and metastatic disease (Cochran $Q=0.01, I^{2}=69 \%$ ).

Adjustment for age differences between arms was examined in individual studies. In one study, patients were significantly older in the arm with low NLR, and it was unclear whether the multivariable model was adjusted for age [9]. In two other studies, the median age in each arm was not reported, and age did not seem to be included in the multivariable model $[10,11]$. In a sensitivity analysis excluding these three studies, high NLR remained a significant predictor for shorter OS (HR 2.55, 95\% CI = $2.59-8.26 ; P<0.001)$. Table 2 presents the results of the meta-regression analysis. We did not identify any classical clinicopathologic factors that were effect modifiers for influence of NLR on OS. Additionally, the median duration of follow-up did not affect the association between high NLR and OS.

There was evidence of publication bias, with fewer smaller studies reporting lower magnitude associations between NLR and OS (Fig. 3).

\section{Disease-free survival}

Nine studies comprising 4864 patients reported HRs for DFS. All studies included only patients with nonmetastatic disease. The median cutoff value for high NLR was 2.5 (range 1.9-4.0). Median length of follow-up was reported in eight studies, ranging from 1.8 to 7.2 years (mean 4.5 years) (Table 4 in Appendix 2). Overall, a NLR greater than the cutoff value was associated with worse DFS (HR 1.74, 95\% CI $=1.47-2.07 ; P<0.001$; see Fig. 2). There was no evidence of statistically significant heterogeneity (Cochran $Q=0.14, I^{2}=35 \%$ ).

Adjustment for age differences between arms was examined in individual studies. Two studies had significant age differences between arms and no clear model adjustment for age, including one study where patients were significantly older in the arm with low NLR [9] and one study where the same group was significantly younger [12]. Another study did not report the median age in each arm and did not adjust for age in the multivariable model [10]. In a sensitivity analysis excluding these three studies, high NLR remained a significant predictor for shorter DFS (HR 1.69, 95\% CI $=1.40-2.03 ; P<0.001$ ).

All studies reported the number of patients with HER2positive disease, while seven of nine studies included data on ER status (Table 4 in Appendix 2). Meta-regression analysis is presented in Table 2. Results showed that ER and HER2 positivity were negative effect modifiers of the association between NLR and DFS, indicating that the NLR has a greater prognostic value in breast cancers that are ER-negative and/or HER2-negative. The proportion of patients with triple-negative or metastatic disease, median age, disease stage, histologic tumor grade, presence of nodal involvement, premenopausal status, median duration of follow-up, and NLR cutoff value did not affect the association between high NLR and DFS. There was evidence of publication bias, with fewer smaller studies reporting lower magnitude associations between NLR and DFS (Fig. 3). 
A

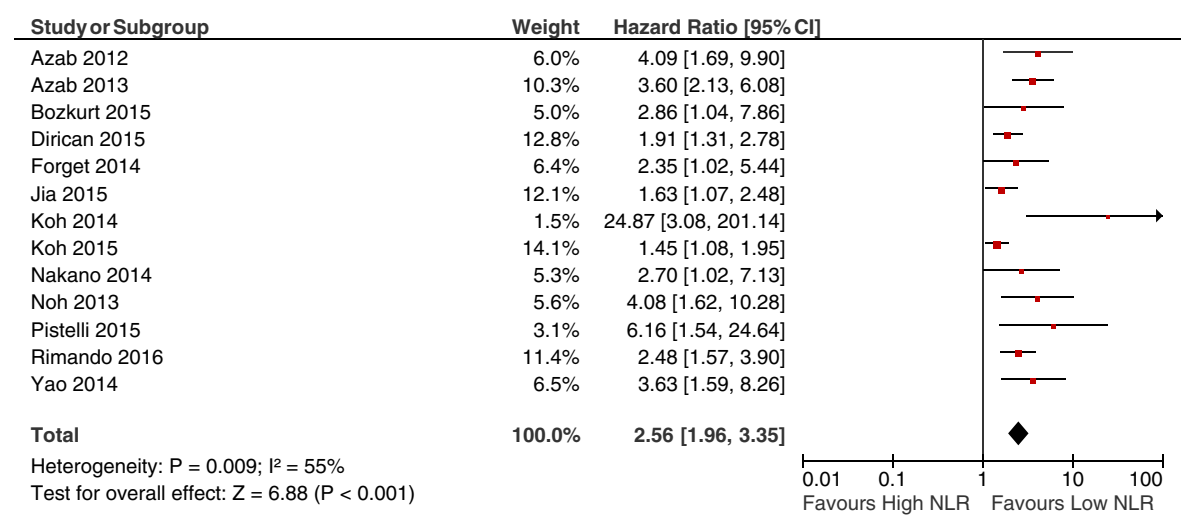

B

\begin{tabular}{|c|c|c|c|c|}
\hline Study or Subgroup & Weight & Hazard Ratio $[95 \% \mathrm{Cl}]$ & & \\
\hline Asano 2016 & $0.2 \%$ & $11.11[0.35,356.75]$ & & \\
\hline Pistelli 2015 & $1.3 \%$ & $5.15[1.11,23.89]$ & & \\
\hline Bozkurt 2015 & $2.0 \%$ & $5.46[1.61,18.52]$ & & \\
\hline Koh 2014 & $4.0 \%$ & $3.87[1.64,9.15]$ & & \\
\hline Nakano 2014 & $5.2 \%$ & $2.00[0.94,4.23]$ & & - \\
\hline Forget 2014 & $10.1 \%$ & $1.99[1.16,3.41]$ & & 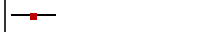 \\
\hline Hong 2015 & $12.7 \%$ & $1.87[1.15,3.02]$ & & -- \\
\hline Dirican 2015 & $25.9 \%$ & $1.46[1.04,2.05]$ & & - \\
\hline Jia 2015 & $38.6 \%$ & $1.50[1.14,1.98]$ & & - \\
\hline Total & $100.0 \%$ & $1.74[1.47,2.07]$ & & $\bullet$ \\
\hline $\begin{array}{l}\text { Heterogeneity: } P=0.14 ; I^{2}=35 \% \\
\text { Test for overall effect: } Z=6.32(P<0.001)\end{array}$ & & & $\begin{array}{lc} & 1 \\
0.01 & 0.1 \\
\text { Favours } & \text { High NLR }\end{array}$ & $\begin{array}{|ccc|} & 1 \\
1 & 10 & 100 \\
\text { Favours Low NLR }\end{array}$ \\
\hline
\end{tabular}

Fig. 2 Forest plots showing HRs for OS (a) and DFS (b) for neutrophil-to-lymphocyte ratio (NLR) greater than or less than the cutoff value. HRs for each study represented by squares: size of the square represents the weight of the study in the meta-analysis, and the horizontal line crossing the square represents the $95 \%$ confidence interval (Cl). All statistical tests were two-sided

\section{Risk of bias assessment}

The risk of bias in individual studies is summarized in Figure 4 in Appendix 3. Overall, risk of bias was low, particularly in the domains of study attrition, prognostic factor measurement, outcome measurement, and statistical analysis and reporting. There was a low-moderate risk of bias for the study participation domain due to lack of completeness in description of the baseline study sample in three studies $[8,13,14]$. Risk of bias was moderate with regards to study confounding, because four studies failed to adequately detail covariates included in adjusted models $[8,10,12,15]$.

\section{Discussion}

High NLR is associated with poor survival in patients diagnosed with several types of cancer [4]. Here we performed a breast cancer-specific meta-analysis, including 15 studies comprising 8563 patients, and found a significant prognostic effect for NLR on both OS and DFS. While there was evidence of publication bias, potentially indicating bias towards publication of positive studies, the overall risk of bias was low, as assessed with the QUIPS tool.
The magnitude of effect on DFS was highest in ERnegative and HER2-negative subtypes. However, this finding does not rule out an effect in ER-positive or HER2-positive subgroups. Rather, the finding indicates a greater magnitude of effect in ER-negative and/or HER2-negative breast cancers. It is possible that the smaller magnitude of effect seen in ER-positive and/or HER2-positive disease relates to the relatively short duration of follow-up of included studies; recurrences occur later in follow-up with ER-positive disease compared with ER-negative disease. However, in a post-hoc meta-regression analysis, median follow-up did not significantly alter the association of NLR with either DFS or OS. Unfortunately, a stratified meta-regression based on ER status was not possible. Some uncertainty therefore remains about the effect of duration of follow-up on subgroups defined by receptor expression.

Despite a greater magnitude of association between NLR and DFS in certain subgroups, patient and disease characteristics did not significantly alter the magnitude of effect of NLR on OS. The negative prognostic effect of NLR on OS was consistent in all clinicopathologic groups and was not influenced by the duration of follow-up in individual studies. One possible explanation 
Table 2 Meta-regression for the association of clinicopathologic factors and the hazard ratio for disease-free and overall survival

\begin{tabular}{|c|c|c|c|}
\hline Variable & Studies included in analysis & Standardized $\beta$ coefficient & $P$ value \\
\hline \multicolumn{4}{|l|}{ Overall survival } \\
\hline Median age & {$[8,9,11,13-15,26-28]$} & 0.098 & 0.80 \\
\hline ER positive & {$[9-11,13,15,23-27]$} & 0.084 & 0.81 \\
\hline HER2 positive & {$[8-11,14,15,23-27]$} & -0.40 & 0.22 \\
\hline Triple negative & {$[8,14,24,27]$} & 0.05 & 0.93 \\
\hline Grade 1 or 2 & {$[8,10,14,15,23-25]$} & 0.02 & 0.95 \\
\hline Grade 3 & {$[8,10,14,15,23-25]$} & -0.02 & 0.95 \\
\hline Stage 0-I & {$[9,13,23,25,27,28]$} & 0.68 & 0.14 \\
\hline Stage II & {$[9,13,23,25,27,28]$} & -0.30 & 0.56 \\
\hline Stage III & {$[9,13,25,27,28]$} & -0.73 & 0.16 \\
\hline Metastatic disease & {$[8-11,13-15,24-28]$} & -0.29 & 0.35 \\
\hline Premenopausal & {$[24,25]$} & 0.04 & 0.95 \\
\hline Nodal involvement & {$[8-11,13-15,23-27]$} & -0.04 & 0.90 \\
\hline NLR cutoff value & {$[8,10,13-15,23,24]$} & -0.29 & 0.33 \\
\hline Median follow-up & {$[8-11,13,14,23,25-28]$} & -0.16 & 0.64 \\
\hline \multicolumn{4}{|l|}{ Disease-free survival } \\
\hline Median age & {$[8,9,14,27,29]$} & 0.06 & 0.93 \\
\hline ER positive & {$[9,10,12,24,25,27,29]$} & -0.77 & $0.04^{*}$ \\
\hline HER2 positive & {$[8-10,12,14,24,25,27,29]$} & -0.79 & $0.01^{*}$ \\
\hline Triple negative & {$[8,12,14,24,27,29]$} & 0.63 & 0.18 \\
\hline Grade 1 or 2 & {$[8-10,12,14,24,25,27,29]$} & -0.46 & 0.21 \\
\hline Grade 3 & {$[8-10,12,14,24,25,27,29]$} & 0.46 & 0.21 \\
\hline Stage $0-1$ & {$[9,25,27,29]$} & 0.46 & 0.54 \\
\hline Stage II & {$[9,25,27,29]$} & 0.53 & 0.36 \\
\hline Stage III & {$[9,25,27,29]$} & -0.50 & 0.39 \\
\hline Metastatic disease & {$[25]$} & -0.74 & 0.49 \\
\hline Premenopausal & {$[9,12,24,25,27]$} & 0.43 & 0.40 \\
\hline Nodal involvement & {$[8-10,12,14,24,25,27,29]$} & 0.25 & 0.52 \\
\hline NLR cutoff value & {$[8-10,12,14,24,25,27,29]$} & -0.15 & 0.70 \\
\hline Median follow-up & {$[8-10,12,14,25,27,29]$} & -0.19 & 0.66 \\
\hline
\end{tabular}

ER estrogen receptor, NLR neutrophil-to-lymphocyte

*Statistically significant at $P<0.05$

for this is that a proportion of breast cancer patients die of causes other than breast cancer, especially cardiovascular disease [16, 17]. Increased NLR has been associated with higher coronary heart disease mortality [18]. The competing risks of cardiovascular and breast cancer deaths may have led to difficulty in exploring the influence of breast cancer-specific characteristics on OS.

While the association between increased NLR and poor outcomes is not fully understood, it has been proposed that high NLR may be indicative of inflammation. In particular, neutrophils have been shown to inhibit the immune system and promote tumor growth by suppressing the activity of lymphocytes and T-cell response [19, 20]. Increased lymphocytic tumor infiltration has also been associated with improved DFS in ER-negative/HER2-negative breast cancer [21]. In our study, we found a greater magnitude of effect on DFS in patients with ER-negative and/or HER2negative disease. However, while this indicates the potential importance of lymphocyte activity, the association between increased tumor-infiltrating lymphocytes and peripheral blood lymphocytes remains unclear. Furthermore, the greater magnitude of association in patients with ERnegative and/or HER2-negative breast cancers was not seen with triple-negative disease. This observation may be due to the relatively small number of studies reporting outcomes in patients with triple-negative breast cancer; 

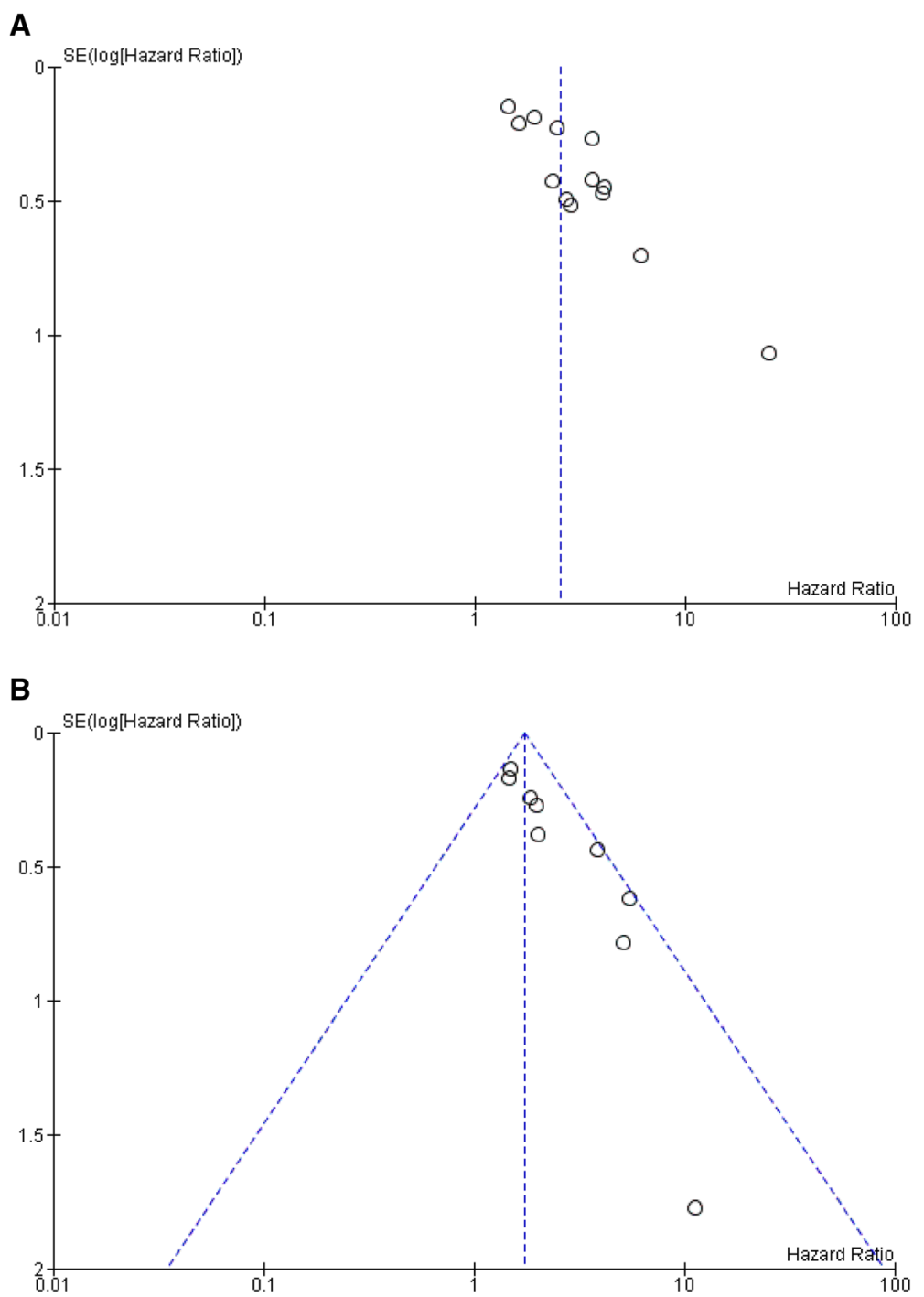

Fig. 3 Funnel plots of HR for OS (a) and DFS (b) for high NLR ratio (horizontal axis) and the standard error (SE) for the HR (vertical axis). Each study is represented by one circle. Vertical line represents the pooled effect estimate

the majority of studies identified patients based on independent subgroups based on ER and HER2 status.

While there are several clinicopathologic factors associated with increased risk of recurrence and/or mortality in patients with breast cancer, the NLR is an inexpensive, readily available prognostic marker, and may allow refinement of risk estimates within disease stages and subgroups. Future studies using NLR in combination with other prognostic markers could potentially identify lower risk patients in whom treatment de-escalation may be appropriate. Furthermore, whether NLR is predictive of response to treatment or provides additional information in cases where risk stratification models exist, such as the 21-gene assay in node-negative ER-positive/HER2-negative disease, is unknown. However, previous research showed no association between NLR and the 21-gene assay recurrence score, indicating that the poor outcomes in patients with high NLR cannot be explained by the proliferation of ER signaling [22]. Further studies examining whether NLR may help refine established prognostic scores are therefore warranted.

\section{Conclusion}

High NLR is associated with an adverse OS and DFS in patients with breast cancer, and its prognostic value is consistent among different clinicopathologic factors such as disease stage and subtype. NLR is an easily accessible prognostic marker, and its addition to established risk prediction models warrants further investigation. 


\section{Appendix 1}

Table 3 Search strategy ${ }^{\mathrm{a}}$

\begin{tabular}{|c|c|c|c|}
\hline Number & Searches & Results & Type \\
\hline 1 & exp Breast Neoplasms/ & 241,242 & Advanced \\
\hline 2 & (breast? adj6 cancer*).mp,kw. & 203,097 & Advanced \\
\hline 3 & (breast? adj6 neoplas*).mp,kw. & 241,382 & Advanced \\
\hline 4 & (breast? adj6 carcin*).mp,kw. & 62,218 & Advanced \\
\hline 5 & (breast? adj6 tumo? ${ }^{*}$ ).mp,kw. & 46,556 & Advanced \\
\hline 6 & (breast? adj6 adenocarcin*).mp,kw. & 4642 & Advanced \\
\hline 7 & (breast? adj6 adeno-carcin*).mp,kw. & 10 & Advanced \\
\hline 8 & (breast? adj6 sarcoma*).mp,kw. & 1271 & Advanced \\
\hline 9 & (breast? adj6 dcis).mp,kw. & 1258 & Advanced \\
\hline 10 & (breast? adj6 ductal).mp,kw. & 16,064 & Advanced \\
\hline 11 & (breast? adj6 infiltrating).mp,kw. & 1418 & Advanced \\
\hline 12 & (breast? adj6 intraductal).mp,kw. & 2294 & Advanced \\
\hline 13 & (breast? adj6 lobular).mp,kw. & 4044 & Advanced \\
\hline 14 & (breast? adj6 medullary).mp,kw. & 383 & Advanced \\
\hline 15 & (breast? adj6 comedo*).mp,kw. & 75 & Advanced \\
\hline 16 & (breast? adj6 metast*).mp,kw. & 26,054 & Advanced \\
\hline 17 & (breast? adj2 malignan*).mp,kw. & 4962 & Advanced \\
\hline 18 & (breast? adj6 onco*).mp,kw. & 3338 & Advanced \\
\hline 19 & (mammar* adj6 cancer*).mp,kw. & 5493 & Advanced \\
\hline 20 & (mammar* adj6 neoplas*).mp,kw. & 21,985 & Advanced \\
\hline 21 & $\left(\right.$ mammar* $^{*}$ adj6 carcin*).mp,kw. & 11,584 & Advanced \\
\hline 22 & $\left(\right.$ mammar* adj6 tumo? $\left.{ }^{*}\right) \cdot m p, k w$. & 18,026 & Advanced \\
\hline 23 & (mammar* adj6 adenocarcin*).mp,kw. & 2958 & Advanced \\
\hline 24 & (mammar* adj6 adeno-carcin*).mp,kw. & 3 & Advanced \\
\hline 25 & (mammar* adj6 sarcoma*).mp,kw. & 384 & Advanced \\
\hline 26 & (mammar* adj6 ductal).mp,kw. & 937 & Advanced \\
\hline 27 & (mammar* adj6 intraductal).mp,kw. & 117 & Advanced \\
\hline 28 & (mammar* adj6 infiltrating).mp,kw. & 201 & Advanced \\
\hline 29 & (mammar* adj6 lobular).mp,kw. & 151 & Advanced \\
\hline 30 & (mammar* adj6 medullary).mp,kw. & 19 & Advanced \\
\hline 31 & (mammar* adj6 comedo*).mp,kw. & 6 & Advanced \\
\hline 32 & (mammar* adj6 metast*).mp,kw. & 2554 & Advanced \\
\hline 33 & (mammar* adj6 malignan*).mp,kw. & 1506 & Advanced \\
\hline 34 & (mammar* adj6 dcis).mp,kw. & 61 & Advanced \\
\hline 35 & (ductal adj6 situ).mp,kw. & 6301 & Advanced \\
\hline 36 & (ductal adj6 carcino*).mp,kw. & 25,790 & Advanced \\
\hline 37 & (paget?? adj6 breast?).mp,kw. & 367 & Advanced \\
\hline 38 & (paget?? adj6 nipple?).mp,kw. & 363 & Advanced \\
\hline 39 & phyllodes.mp,kw. & 1876 & Advanced \\
\hline 40 & phylloides.mp,kw. & 206 & Advanced \\
\hline 41 & cystosarcoma*.mp,kw. & 603 & Advanced \\
\hline 42 & DCIS.mp,kw. & 3401 & Advanced \\
\hline 43 & or/1-40 & 318,397 & Advanced \\
\hline 44 & exp Ovarian Neoplasms/ & 71,707 & Advanced \\
\hline 45 & (ovar* adj6 cancer*).mp,kw. & 44,037 & Advanced \\
\hline
\end{tabular}


Table 3 Search strategy ${ }^{a}$ (Continued)

\begin{tabular}{|c|c|c|c|}
\hline 46 & (ovar* adj6 neoplas*).mp,kw. & 71,929 & Advanced \\
\hline 47 & (ovar* adj6 tumo?r*).mp,kw. & 24,113 & Advanced \\
\hline 48 & (ovar* adj6 malignan*).mp,kw. & 7601 & Advanced \\
\hline 49 & (ovar* adj6 metasta*).mp,kw. & 5781 & Advanced \\
\hline 50 & $\left(o v a r^{*} \operatorname{adj} 6 \operatorname{carcin}^{*}\right) \cdot m p, k w$. & 18,742 & Advanced \\
\hline 51 & (ovar* adj6 adenocarcin*).mp,kw. & 2966 & Advanced \\
\hline 52 & (ovar* adj6 adeno-carcin*).mp,kw. & 12 & Advanced \\
\hline 53 & (ovar* adj6 choriocarcin*).mp,kw. & 217 & Advanced \\
\hline 54 & (granulosa adj6 cancer*).mp,kw. & 54 & Advanced \\
\hline 55 & (granulosa adj6 tumo?r*).mp,kw. & 2699 & Advanced \\
\hline 56 & (granulosa adj6 neoplas*).mp,kw. & 173 & Advanced \\
\hline 57 & (granulosa adj6 malignan*).mp,kw. & 142 & Advanced \\
\hline 58 & (granulosa adj6 metasta*).mp,kw. & 111 & Advanced \\
\hline 59 & (granulosa adj6 carcin*).mp,kw. & 118 & Advanced \\
\hline 60 & (granulosa adj6 adenocarcin*).mp,kw. & 45 & Advanced \\
\hline 61 & (granulosa adj6 adeno-carcin*).mp,kw. & 0 & Advanced \\
\hline 62 & OGCTs.mp,kw. & 28 & Advanced \\
\hline 63 & HBOC.mp,kw. & 650 & Advanced \\
\hline 64 & Luteoma*.mp,kw. & 203 & Advanced \\
\hline 65 & Sertoli-Leydig*.mp,kw. & 1039 & Advanced \\
\hline 66 & Thecoma*.mp,kw. & 1013 & Advanced \\
\hline 67 & (theca* adj6 tumo? ${ }^{*}$ ).mp,kw. & 493 & Advanced \\
\hline 68 & (ovar* adj6 dysgerminoma?).mp,kw. & 467 & Advanced \\
\hline 69 & androblastoma*.mp,kw. & 321 & Advanced \\
\hline 70 & arrhenoblastoma*.mp,kw. & 349 & Advanced \\
\hline 71 & arrheno-blastoma*.mp,kw. & 1 & Advanced \\
\hline 72 & Meig*.mp,kw. & 2152 & Advanced \\
\hline 73 & or/44-72 & 93,590 & Advanced \\
\hline 74 & exp Endometrial Neoplasms/ & 17,416 & Advanced \\
\hline 75 & (endometr* adj6 neoplas*).mp,kw. & 17,866 & Advanced \\
\hline 76 & (endometr* adj6 cancer*).mp,kw. & 15,307 & Advanced \\
\hline 77 & (endometr* adj6 tumo?r*).mp,kw. & 5128 & Advanced \\
\hline 78 & (endometr* adj6 carcino*).mp,kw. & 12,730 & Advanced \\
\hline 79 & (endometr* adj6 adenocarcin*).mp,kw. & 5361 & Advanced \\
\hline 80 & (endometr* adj6 adeno-carcin*).mp,kw. & 9 & Advanced \\
\hline 81 & (endometr* adj6 sarcoma*).mp,kw. & 1230 & Advanced \\
\hline 82 & (endometr* adj6 malignan*).mp,kw. & 2300 & Advanced \\
\hline 83 & (endometr* adj6 metast*).mp,kw. & 1337 & Advanced \\
\hline 84 & (endometr* adj6 onco*).mp,kw. & 370 & Advanced \\
\hline 85 & (endometr* adj6 choriocarcin*).mp,kw. & 88 & Advanced \\
\hline 86 & or/74-85 & 31,774 & Advanced \\
\hline 87 & Uterine Cervical Neoplasms/ & 65,130 & Advanced \\
\hline 88 & (cervi* adj6 cancer*).mp,kw. & 41,277 & Advanced \\
\hline 89 & (cervi* adj6 neoplas*).mp,kw. & 69,153 & Advanced \\
\hline 90 & $\left(\right.$ cervi* adj6 tumo? $\left.r^{*}\right) \cdot m p, k w$. & 7715 & Advanced \\
\hline 91 & (cervi* adj6 malignan*).mp,kw. & 3006 & Advanced \\
\hline 92 & (cervi* adj6 metast*).mp,kw. & 6612 & Advanced \\
\hline
\end{tabular}


Table 3 Search strategy ${ }^{a}$ (Continued)

\begin{tabular}{|c|c|c|c|}
\hline 93 & (cervi* adj6 onco*).mp,kw. & 1280 & Advanced \\
\hline 94 & $($ cervi* adj6 carcin*).mp,kw. & 24,588 & Advanced \\
\hline 95 & (cervi* adj6 adenocarcin*).mp,kw. & 2945 & Advanced \\
\hline 96 & (cervi* adj6 adeno-carcin*).mp,kw. & 9 & Advanced \\
\hline 97 & (cervi* adj6 squamous*).mp,kw. & 7833 & Advanced \\
\hline 98 & (cervi* adj6 adenosquamous*).mp,kw. & 211 & Advanced \\
\hline 99 & (cervi* adj6 adeno-squamous*).mp,kw. & 2 & Advanced \\
\hline 100 & $($ cervi* adj6 sarcoma*).mp,kw. & 661 & Advanced \\
\hline 101 & (cervi* adj6 small cell*).mp,kw. & 364 & Advanced \\
\hline 102 & (cervi* adj6 large cell*).mp,kw. & 78 & Advanced \\
\hline 103 & (cervi* adj6 neuroendocrine*).mp,kw. & 195 & Advanced \\
\hline 104 & (cervi* adj6 neuro-endocrine*).mp,kw. & 2 & Advanced \\
\hline 105 & (cervi* adj6 choriocarcin*).mp,kw. & 112 & Advanced \\
\hline 106 & scCC.mp,kw. & 46 & Advanced \\
\hline 107 & or/87-106 & 90,890 & Advanced \\
\hline 108 & 73 or 86 or 107 & 199,155 & Advanced \\
\hline 109 & exp Lymphocytes/ & 461,529 & Advanced \\
\hline 110 & lymphocyte?.mp,kw. & 554,948 & Advanced \\
\hline 111 & (lymphoid adj2 cell?).mp,kw. & 22,666 & Advanced \\
\hline 112 & (killer adj4 cell?).mp,kw. & 51,337 & Advanced \\
\hline 113 & (nk adj2 cell?).mp,kw. & 31,413 & Advanced \\
\hline 114 & (lak adj2 cell?).mp,kw. & 2650 & Advanced \\
\hline 115 & b-lymphocyte?.mp,kw. & 93,264 & Advanced \\
\hline 116 & t-lymphocyte?.mp,kw. & 290,882 & Advanced \\
\hline 117 & b-lymphoid.mp,kw. & 2219 & Advanced \\
\hline 118 & t-lymphoid.mp,kw. & 1196 & Advanced \\
\hline 119 & (plasm adj2 cell?).mp,kw. & 31 & Advanced \\
\hline 120 & plasmacyte?.mp,kw. & 341 & Advanced \\
\hline 121 & (immune adj3 cell?).mp,kw. & 58,743 & Advanced \\
\hline 122 & (immunocompetent adj2 cell?).mp,kw. & 3494 & Advanced \\
\hline 123 & immnunocyte?.mp,kw. & 0 & Advanced \\
\hline 124 & immnuno-cyte?.mp,kw. & 0 & Advanced \\
\hline 125 & lymph cell?.mp,kw. & 184 & Advanced \\
\hline 126 & null cell?.mp,kw. & 3404 & Advanced \\
\hline 127 & immunological* competent cell?.mp,kw. & 153 & Advanced \\
\hline 128 & immunoreactive cell?.mp,kw. & 6231 & Advanced \\
\hline 129 & immuno-reactive cell?.mp,kw. & 18 & Advanced \\
\hline 130 & prolymphocyte?.mp. & 218 & Advanced \\
\hline 131 & pro-lymphocyte?.mp. & 3 & Advanced \\
\hline 132 & or/109-131 & 648,538 & Advanced \\
\hline 133 & Neutrophils/ & 77,202 & Advanced \\
\hline 134 & neutrophil*.mp,kw. & 135,327 & Advanced \\
\hline 135 & (cell? adj2 le).mp,kw. & 868 & Advanced \\
\hline 136 & (leukocyte? adj3 polymorphonuclear).mp,kw. & 14,471 & Advanced \\
\hline 137 & pmn granulocyte?.mp,kw. & 52 & Advanced \\
\hline 138 & pmn leukocyte?.mp,kw. & 400 & Advanced \\
\hline 139 & (poly morphou* adj2 granulocyte?).mp,kw. & 0 & Advanced \\
\hline
\end{tabular}


Table 3 Search strategy ${ }^{a}$ (Continued)

\begin{tabular}{|c|c|c|c|}
\hline 140 & (polynuclear adj3 leukocyte?).mp,kw. & 71 & Advanced \\
\hline 141 & or/133-140 & 139,999 & Advanced \\
\hline 142 & (neutrophil? adj6 lymphocyte?).mp,kw. & 8790 & Advanced \\
\hline 143 & NLR.mp,kw. & 1729 & Advanced \\
\hline 144 & 132 and 141 & 26,722 & Advanced \\
\hline 145 & or/142-144 & 27,810 & Advanced \\
\hline 146 & exp Cohort Studies/ & $1,522,637$ & Advanced \\
\hline 147 & exp Prognosis/ & $1,240,142$ & Advanced \\
\hline 148 & exp Morbidity/ & 425,952 & Advanced \\
\hline 149 & exp Mortality/ & 309,548 & Advanced \\
\hline 150 & exp survival analysis/ & 214,369 & Advanced \\
\hline 151 & exp models, statistical/ & 311,009 & Advanced \\
\hline 152 & prognos*.mp,kw. & 603,945 & Advanced \\
\hline 153 & predict*.mp,kw. & $1,026,266$ & Advanced \\
\hline 154 & course*.mp,kw. & 467,535 & Advanced \\
\hline 155 & diagnosed.mp,kw. & 361,373 & Advanced \\
\hline 156 & cohort*.mp,kw. & 388,862 & Advanced \\
\hline 157 & death?.mp,kw. & 646,834 & Advanced \\
\hline 158 & or/146-157 & $4,572,550$ & Advanced \\
\hline 159 & 108 and 145 and 158 & 64 & Advanced \\
\hline 160 & 43 and 145 and 158 & 122 & Advanced \\
\hline 161 & 159 or 160 & 184 & Advanced \\
\hline 162 & limit 161 to yr = "2013-Current" & 85 & Advanced \\
\hline
\end{tabular}

${ }^{\text {aOvid MEDLINE }}$, 1946-April week 22016

\section{Appendix 2}

Table 4 Detailed characteristics of included studies

\begin{tabular}{|c|c|c|c|c|c|c|c|c|c|c|c|c|}
\hline \multirow[t]{2}{*}{ Author } & \multirow[t]{2}{*}{ Year } & \multirow{2}{*}{$\begin{array}{l}\text { Number } \\
\text { of patients }\end{array}$} & \multirow{2}{*}{$\begin{array}{l}\text { Disease } \\
\text { stage }\end{array}$} & \multirow{2}{*}{$\begin{array}{l}\text { NLR } \\
\text { cutoff } \\
\text { value }\end{array}$} & \multirow{2}{*}{$\begin{array}{l}\text { Median } \\
\text { age } \\
\text { (years) }\end{array}$} & \multicolumn{3}{|c|}{ Breast cancer subtype (\%) } & \multicolumn{2}{|l|}{ Grade (\%) } & \multirow[t]{2}{*}{ Postmenopausal (\%) } & \multirow{2}{*}{$\begin{array}{l}\text { Median } \\
\text { follow-up } \\
\text { (years) }\end{array}$} \\
\hline & & & & & & $\begin{array}{l}\text { ER } \\
+\end{array}$ & $\begin{array}{l}\text { HER-2 } \\
+\end{array}$ & $\begin{array}{l}\text { Triple } \\
\text { negative }\end{array}$ & $\begin{array}{l}\text { Grade 1- } \\
2\end{array}$ & $\begin{array}{l}\text { Grade } \\
3\end{array}$ & & \\
\hline Asano et al. [12] & 2016 & 61 & Early & 3.0 & $\mathrm{n} / \mathrm{a}$ & 0 & 0 & 100 & 72 & 28 & 36 & 3.1 \\
\hline Azab et al. [23] & 2012 & 316 & Mixed & 3.3 & $\mathrm{n} / \mathrm{a}$ & 83 & 17 & $\mathrm{n} / \mathrm{a}$ & 70 & 30 & $\mathrm{n} / \mathrm{a}$ & 3.8 \\
\hline Azab et al. [13] & 2013 & 437 & Mixed & 3.3 & 64 & 76 & $\mathrm{n} / \mathrm{a}$ & $\mathrm{n} / \mathrm{a}$ & $\mathrm{n} / \mathrm{a}$ & $\mathrm{n} / \mathrm{a}$ & $\mathrm{n} / \mathrm{a}$ & 5 \\
\hline Bozkurt et al. [24] & 2015 & 85 & Early & 2.0 & $\mathrm{n} / \mathrm{a}$ & 0 & 0 & 100 & 31 & 69 & 69 & $n / a$ \\
\hline Dirican et al. [25] & 2015 & 1527 & Mixed & 4.0 & $\mathrm{n} / \mathrm{a}$ & 68 & 17 & $\mathrm{n} / \mathrm{a}$ & 80 & 20 & 44 & 2.5 \\
\hline Forget et al. [10] & 2014 & 720 & Early & 3.3 & $\mathrm{n} / \mathrm{a}$ & 84 & 9 & $\mathrm{n} / \mathrm{a}$ & 61 & 39 & $\mathrm{n} / \mathrm{a}$ & 5.8 \\
\hline Hong et al. [29] & 2015 & 487 & Early & 1.9 & 55 & 67 & 21 & 19 & 73 & 27 & 42 & 4.6 \\
\hline Jia et al. [14] & 2015 & 1570 & Early & 2.0 & 47 & $\mathrm{n} / \mathrm{a}$ & 22 & 14 & 62 & 38 & $\mathrm{n} / \mathrm{a}$ & 6.6 \\
\hline Koh et al. [8] & 2014 & 157 & Early & 2.3 & 44 & $\mathrm{n} / \mathrm{a}$ & 0 & 0 & 80 & 20 & $\mathrm{n} / \mathrm{a}$ & 1.8 \\
\hline Koh et al. [15] & 2015 & 1435 & Mixed & 5.0 & 52 & 55 & 36 & 100 & 56 & 44 & $\mathrm{n} / \mathrm{a}$ & $\mathrm{n} / \mathrm{a}$ \\
\hline Nakano et al. [9] & 2015 & 167 & Early & 2.5 & 58 & 78 & 18 & $\mathrm{n} / \mathrm{a}$ & 80 & 20 & 25 & $7.2^{\mathrm{a}}$ \\
\hline Noh et al. [26] & 2013 & 442 & Early & 2.5 & 50 & 71 & 29 & 18 & 71 & 29 & $\mathrm{n} / \mathrm{a}$ & 5.9 \\
\hline Pistelli et al. [27] & 2015 & 90 & Early & 3.0 & 53 & 0 & 0 & 100 & 10 & 90 & 40 & 4.5 \\
\hline Rimando et al. [28] & 2016 & 461 & Mixed & 3.8 & 58 & 74 & $\mathrm{n} / \mathrm{a}$ & $\mathrm{n} / \mathrm{a}$ & 51 & 49 & $\mathrm{n} / \mathrm{a}$ & 5.1 \\
\hline Yao et al. [11] & 2014 & 608 & Early & 2.6 & 53 & 66 & 25 & 16 & $\mathrm{n} / \mathrm{a}$ & $\mathrm{n} / \mathrm{a}$ & 48 & 3.5 \\
\hline
\end{tabular}

$E R$ estrogen receptor, $n / a$ not available, $N L R$ neutrophil-to-lymphocyte 


\section{Appendix 3}

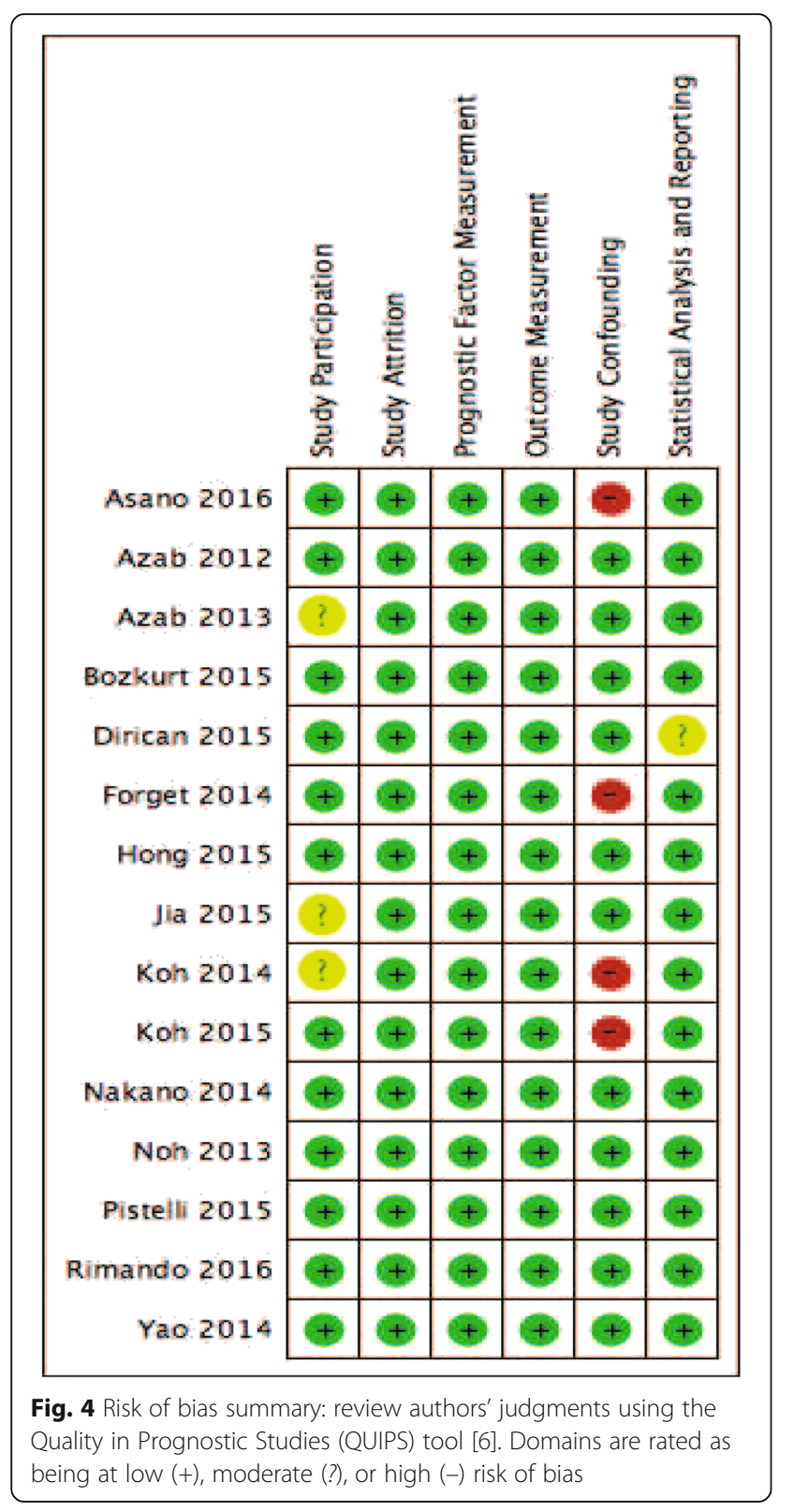

\section{Abbreviations}

Cl: Confidence interval; DFS: Disease-free survival; ER: Estrogen receptor; HR: Hazard ratio; NLR: Neutrophil-to-lymphocyte ratio; OS: Overall survival; PFS: Progression-free survival; PR: Progesterone receptor; SE: Standard error

\section{Acknowledgements}

The authors wish to thank Rouhi Fazelzad for conducting the literature search.

\section{Funding}

No funding was received.

\section{Availability of data and materials}

Detailed characteristics of included studies are presented in Table 4 in Appendix 2.

\section{Authors' contributions}

J-LE collected, analyzed, and interpreted the data and was a major contributor in writing the manuscript. DD was the second reviewer for data collection, analysis, and risk of bias assessment. EA, AT, and PSS also participated in data analysis and interpretation, as well as manuscript preparation. All authors read and approved the final manuscript.

\section{Competing interests}

The authors declare that they have no conflicts of interest.

\section{Consent for publication}

Not applicable. Literature reviews and meta-analyses do not require patient consent for publication in Canada.

Ethics approval and consent to participate

Not applicable. Literature reviews and meta-analyses do not require ethics approval in Canada.

\section{Author details}

'Division of Medical Oncology and Hematology, Princess Margaret Cancer Centre, 610 University Avenue 5-124, Toronto, ON M5G 2M9, Canada. ${ }^{2}$ Department of Medicine, University of Toronto, Toronto, Canada. ${ }^{3}$ Division of Medical Oncology and Hematology, Sunnybrook Health Sciences Centre University of Toronto, Toronto, Canada. ${ }^{4}$ Department of Medical Oncology, St. Claraspital Basel and Faculty of Medicine, University of Basel, Basel, Switzerland. ${ }^{5}$ Institute of Health Policy and Management Evaluation, University of Toronto, Toronto, Canada. ${ }^{6}$ Department of Paediatrics, Mount Sinai Hospital, University of Toronto, Toronto, Canada.

Received: 19 September 2016 Accepted: 6 December 2016

Published online: 05 January 2017

\section{References}

1. Masood S. Prognostic/predictive factors in breast cancer. Clin Lab Med. 2005;25:809-25.

2. Hanahan D, Weinberg R. Hallmarks of cancer: the next generation. Cell. 2011;144:646-74.

3. Guthrie GJK, Charles KA, Roxburgh CSD, Horgan PG, McMillan DC, Clarke SJ. The systemic inflammation-based neutrophil-lymphocyte ratio: experience in patients with cancer. Crit Rev Oncol Hematol. 2013;88(1):218-30. http://dx.doi.org/10.1016/j.critrevonc.2013.03.010.

4. Templeton AJ, McNamara MG, Seruga B, Vera-Badillo FE, Aneja P, Ocana A, Leibowitz-Amit R, Sonpavde G, Knox JJ, Tran B, Tannock IF, Amir E. Prognostic role of neutrophil-to-lymphocyte ratio in solid tumors: a systematic review and meta-analysis. J Natl Cancer Inst. 2014;106(6):dju124. doi:10.1093/jnci/dju124.

5. Liberati A, Altman D, Tetzlaff J. The PRISMA statement for reporting systematic reviews and meta-analyses of studies that evaluate health care interventions: explanation and elaboration. PLoS Med. 2009;6(7).

6. Hayden J, van der Windt D, Cartwright J, Côté P, Bombardier C. Assessing bias in studies of prognostic factors. Ann Intern Med. 2013;158(4):280-6.

7. Deeks J, Higgins J, Altman D. Chapter 9: Analysing data and under-taking meta-analyses. In: Higgins JPT, Green S, Higgins JPT, Green S, editors. Cochrane Handbook for Systematic Reviews of Interventions Version 5.1.0 (updated March 2011). The Cochrane Collaboration, John Wiley \& Sons; 2011

8. Koh YW, Lee HJ, Ahn JH, Lee JW, Gong G. Prognostic significance of the ratio of absolute neutrophil to lymphocyte counts for breast cancer patients with ER/PR-positivity and HER2-negativity in neoadjuvant setting. Tumour Biol. 2014;35(10):9823-30. http://dx.doi.org/10.1007/s13277-014-2282-5.

9. Nakano K, Hosoda M, Yamamoto M, Yamashita H. Prognostic significance of pre-treatment neutrophil: Iymphocyte ratio in Japanese patients with breast cancer. Anticancer Res. 2014;34(7):3819-24.

10. Forget P, Bentin C, Machiels JP, Berliere M, Coulie PG, De Kock M Intraoperative use of ketorolac or diclofenac is associated with improved disease-free survival and overall survival in conservative breast cancer surgery. Br J Anaesth. 2014;113 Suppl 1:82-7. http://dx.doi.org/10.1093/bja/aet464.

11. Yao M, Liu Y, Jin H, Liu X, Lv K, Wei H, Du C, Wang S, Wei B, Fu P. Prognostic value of preoperative inflammatory markers in Chinese patients with breast cancer. OncoTargets and therapy. 2014;7:1743-52. http://dx.doi.org/10.2147/OTT.S69657. 
12. Asano Y, Kashiwagi S, Onoda N, Noda S, Kawajiri H, Takashima T, Ohsawa M, Kitagawa S, Hirakawa K. Predictive value of neutrophil/lymphocyte ratio for efficacy of preoperative chemotherapy in triple-negative breast cancer. Ann Surg Oncol. 2016;23(4):1104-10. http://dx.doi.org/10.1245/s10434-015-4934-0.

13. Azab B, Shah N, Radbel J, Tan P, Bhatt V, Vonfrolio S, Habeshy A, Picon A, Bloom S. Pretreatment neutrophil/lymphocyte ratio is superior to platelet/lymphocyte ratio as a predictor of long-term mortality in breast cancer patients. Med Oncol. 2013;30(1):432. http://dx.doi.org/10.1007/s12032-012-0432-4.

14. Jia W, Wu J, Jia H, Yang Y, Zhang X, Chen K, Su F. The peripheral blood neutrophil-to-lymphocyte ratio is superior to the lymphocyte-to-monocyte ratio for predicting the long-term survival of triple-negative breast cancer patients. PLOS ONE. 2015;10(11):e0143061. http://dx.doi.org/10.1371/journal.pone.0143061.

15. Koh CH, Bhoo-Pathy N, Ng KL, Jabir RS, Tan GH, See MH, Jamaris S, Taib NA. Utility of pre-treatment neutrophil-lymphocyte ratio and plateletlymphocyte ratio as prognostic factors in breast cancer. Br J Cancer. 2015; 113(1):150-8. http://dx.doi.org/10.1038/bjc.2015.183.

16. Patnaik J, Byers T, DiGuiseppi C, Dabelea D, Denberg T. Cardiovascular disease competes with breast cancer as the leading cause of death for older females diagnosed with breast cancer: a retrospective cohort study. Breast Cancer Res. 2011;13(3):R64.

17. Abdel-Qadir H, Austin PC, Lee DS, et al. A population-based study of cardiovascular mortality following early-stage breast cancer. JAMA Cardiol. 2016. doi:10.1001/jamacardio.2016.3841.

18. Shah N, Parikh V, Patel N, Patel N, Badheka A, Deshmukh A, Rathod A, Lafferty J. Neutrophil lymphocyte ratio significantly improves the Framingham risk score in prediction of coronary heart disease mortality: insights from the National Health and Nutrition Examination Survey-III. Int J Cardiol. 2014;17(3):390-7.

19. De Larco J, Wuertz B, Furcht L. The potential role of neutrophils in promoting the metastatic phenotype of tumors releasing interleukin-8. Clin Cancer Res. 2004;10(4):895-900.

20. El-Hag A, Clark R. Immunosuppression by activated human neutrophils. Dependence on the myeloperoxidase system. J Immunol. 1987;139(7):2406-13.

21. Loi S, Sirtaine N, Piette F, Salgado R, Viale G, Van Eenoo F, Rouas G, Francis P, Crown JP, Hitre E, de Azambuja E, Quinaux E, Di Leo A, Michiels S, Piccart MJ, Sotiriou C. Prognostic and predictive value of tumor-infiltrating lymphocytes in a phase III randomized adjuvant breast cancer trial in nodepositive breast cancer comparing the addition of docetaxel to doxorubicin with doxorubicin-based chemotherapy: BIG 02-98. J Clin Oncol. 2013;31(7): 860-7. doi:10.1200/JCO.2011.41.0902.

22. Srikanthan A, Bedard PL, Goldstein S, Templeton A, Amir E. Association between the neutrophil-to-lymphocyte ratio (NLR) and the 21-gene recurrence score. Cancer Research Conference: 38th Annual CTRC AACR San Antonio Breast Cancer Symposium San Antonio, TX, USA. Conference Start. 2016;76(4 Suppl 1). doi: http://dx.doi.org/10.1158/1538-7445.SABCS15-P2-08-05

23. Azab B, Bhatt VR, Phookan J, Murukutla S, Kohn N, Terjanian T, Widmann WD. Usefulness of the neutrophil-to-lymphocyte ratio in predicting short- and longterm mortality in breast cancer patients. Ann Surg Oncol. 2012;19(1):217-24. http://dx.doi.org/10.1245/s10434-011-1814-0.

24. Bozkurt O, Karaca H, Berk V, Inanc M, Duran AO, Ozaslan E, Ucar M, Ozkan M. Predicting the role of the pretreatment neutrophil to lymphocyte ratio in the survival of early triple-negative breast cancer patients. J BUON. 2015;20(6):1432-9.

25. Dirican A, Kucukzeybek BB, Alacacioglu A, Kucukzeybek Y, Erten C, Varol U, Somali I, Demir L, Bayoglu IV, Yildiz Y, Akyol M, Koyuncu B, Coban E, Ulger E, Unay FC, Tarhan MO. Do the derived neutrophil to lymphocyte ratio and the neutrophil to lymphocyte ratio predict prognosis in breast cancer? Int J Clin Oncol. 2015;20(1):70-81. http://dx.doi.org/10.1007/s10147-014-0672-8.

26. Noh H, Eomm M, Han A. Usefulness of pretreatment neutrophil to lymphocyte ratio in predicting disease-specific survival in breast cancer patients. J Breast Cancer. 2013;16(1):55-9. http://dx.doi.org/10.4048/jbc.2013.16.1.55.

27. Pistelli M, De Lisa M, Ballatore Z, Caramanti M, Pagliacci A, Battelli N, Santinelli A, Biscotti T, Berardi R, Cascinu S. Pretreatment neutrophil to lymphocyte ratio may be an useful tool in predicting survival in early triplenegative breast cancer patients. J Clin Oncol. 2014;32(15 Suppl 1):195.
28. Rimando J, Campbell J, Kim JH, Tang SC, Kim S. The pretreatment neutrophil/lymphocyte ratio is associated with all-cause mortality in black and white patients with non-metastatic breast cancer. Front Oncol. 2016; 6(81). http://dx.doi.org/10.3389/fonc.2016.00081

29. Hong J, Mao Y, Chen X, Zhu L, He J, Chen W, Li Y, Lin L, Fei X, Shen K. Elevated preoperative neutrophil-to-lymphocyte ratio predicts poor diseasefree survival in Chinese women with breast cancer. Tumour Biol. 2015;21:21. http://dx.doi.org/10.1007/s13277-015-4233-1.

\section{Submit your next manuscript to BioMed Central and we will help you at every step:}

- We accept pre-submission inquiries

- Our selector tool helps you to find the most relevant journal

- We provide round the clock customer support

- Convenient online submission

- Thorough peer review

- Inclusion in PubMed and all major indexing services

- Maximum visibility for your research

Submit your manuscript at www.biomedcentral.com/submit
Ciomed Central 